04

\title{
Инициирование высоковольтным электрическим разрядом взрывчатых превращений в энергонасыщенных материалах с наноразмерными добавками
}

\author{
(C) Г.Г. Савенков, ${ }^{1,4}$ С.А. Рашковский, ${ }^{2}$ В.А. Морозов, ${ }^{3}$ А.А. Лукин, ${ }^{3}$ И.А. Оськин, ${ }^{1}$ \\ B.A. Брагин, ${ }^{1}$ A.В. Шамильянов ${ }^{4}$ \\ ${ }^{1}$ Научно-производственное объединение „Поиск“, \\ 195197 Мурино Ленинградской обл., Россия \\ ${ }^{2}$ Институт проблем механики им. Ишлинского РАН, \\ 119526 Москва, Россия \\ ${ }^{3}$ Санкт-Петербургский государственный университет, \\ 199034 Санкт-Петербург, Россия \\ ${ }^{4}$ Санкт-Петербургский государственный технологический институт (Технический университет), \\ 190013 Санкт-Петербург, Россия \\ e-mail: sav-georgij@yandex.ru
}

(Поступило в Редакцию 29 ноября 2016 г.)

Представлены результаты экспериментальных исследований влияния наноразмерных инертных добавок на пороговые характеристики напряжения пробоя (инициирования) в энергонасыщенных материалах. Предложена физико-математическая модель, описывающая полученные результаты экспериментов.

DOI: 10.21883/JTF.2017.09.44906.2112

\section{Введение}

Инициирование энергонасыщенных материалов (ЭНМ) (в частности, взрывчатых веществ (ВВ)) при помощи высоковольтного электрического разряда достаточно давно реализовано в ряде устройств, например, в так называемых искровых электродетонаторах [1]. Возбуждение взрывчатых превращений (горения и детонации) в таких устройствах происходит за счет высоких значений давления и температуры в искровом (плазменном) канале, образующемся в результате электрического пробоя в ВВ, которое, как правило, является диэлектриком [2].

Преимущество такого способа инициирования ВВ высокое быстродействие (несколько $\mu \mathrm{s}$ ), недостаток (на сегодняшний день) - высокие значения импульса напряжения. В работе [3] были проведены экспериментальные исследования, направленные на оценку влияния проводящего наноразмерного порошка на чувствительность к высоковольтному электрическому разряду энергонасыщенной (взрывчатой) композиции малочувствительного высокодисперсного (средний размер частиц $\sim 5 \mu \mathrm{m}$ ) взрывчатого вещества FOX-7 с указанным порошком. Было установлено, что 15\% массовой доли нанопорошка меди снижают порог инициирования (который, естественно, совпадает с электрической прочностью) с $15 \mathrm{kV}$ до $7.5-9.0 \mathrm{kV}$. В работе [4] на основе экспериментальных данных [3] были разработаны модель поверхностной перколяции электрического пробоя и модель возбуждения детонации в бризантном ВВ (диэлектрике) с наноразмерными металлическими добавками.

Положительная роль наноразмерных инертных добавок в энергонасыщенные материалы при их инициирова- нии сильноточным электронным пучком обсуждалась в работах $[5,6]$.

Однако проведенные исследования в [3] и [4] касались по большей части металлических (медных) наноразмерных порошков, которые имеют повышенную способность к агломерированию и, как все металлические наноразмерные порошки, со временем покрываются окисной пленкой, теряя при этом свои электропроводящие свойства. Кроме того, эксперименты проводились с высокодисперсным ВВ, что в принципе уменьшает вероятность электрического пробоя по воздуху в порах, размер которых соизмерим с размерами частиц ВВ. И наконец, в указанных работах эксперименты проводились при параллельном расположении электродов.

Что же будет с пороговыми значениями напряжения инициирования (электрической прочности) когда размер частиц ЭНМ будет, по крайней мере, на порядок превышать размер частиц работы [3], когда наноразмерные добавки будут являться полупроводящими или диэлектриками, но с меньшей электрической прочностью, чем основное энергонасыщенное вещество, и когда электроды будут установлены друг против друга? Цель настоящей работы - ответы на поставленные вопросы.

\section{Экспериментальная часть}

\section{Объекты исследования и экспериментальная методика}

В качестве энергонасыщенных материалов были выбраны два состава: на основе пикрата калия (в дальнейшем - ЭНМ-1) и на основе свинцового сурика (ЭНМ-2). Размер частиц для обоих ЭНМ 100 $\mu \mathrm{m}$. 
В качестве наноразмерных добавок были выбраны следующие порошки.

1.Наноразмерный медный порошок с первоначальными размерами 50-70 nm, но покрытый толстой окисной пленкой толщиной 50-60 nm, так что порошок был абсолютно черного цвета с размерами частиц 100-130 nm (в дальнейшем будем обозначать этот порошок - $\mathrm{CuO}$ ).

2. Детонационные наноалмазы (ДНА), размер частиц которых менее $100 \mathrm{~nm}$.

Выбор второй добавки определялся тем, что ДНА являются диэлектриками и представляют собой полирадикалы, которые гипотетически должны катализировать цепные процессы при горении энергонасыщенных материалов. И, кроме того, ДНА более химически инертны, чем металлические наноразмерные порошки, и поэтому можно не опасться, что со временем они покроются окисной пленкой. Стоит отметить, что применение ДНА в качестве гипотетической добавки в ЭНМ (по крайней мере, в относящиеся к взрывчатым веществам и составам), которые могут повысить те или иные эксплуатационные характеристики ЭНМ, пока практически не рассматривается.

Смесь ЭНМ-1 с $\mathrm{CuO}$ или с ДНА перемешивалась до получения однородного цвета и помещалась в контейнер из оргстекла (диэлектрик) (рис. 1), аналогично получали композицию ЭНМ-2 с CuO. Полученные таким образом заряды имели практически насыпную плотность.

Внешний диаметр корпуса контейнера $d_{1}=20 \mathrm{~mm}$, внутренний диаметр $d_{2}=10 \mathrm{~mm}$, толщина стенок и донышка контейнера $h_{1}=5 \mathrm{~mm}$. Глубина контейнера $\left(h_{2}+\right.$ $\left.+h_{3}\right)=10 \mathrm{~mm}$, на высоте $h_{2}=5 \mathrm{~mm}$ от дна корпуса в стенке корпуса выполнены два отверстия под стальные остроконечные электроды диаметром $3 \mathrm{~mm}$. Корпус контейнера закрывается крышкой из оргстекла толщиной $h_{4}=5 \mathrm{~mm}$, которая крепится к корпусу двумя винтами. Расстояние между электродами $a=3 \mathrm{~mm}$. Электрическая схема проведения эксперимента представлена на рис. 2.

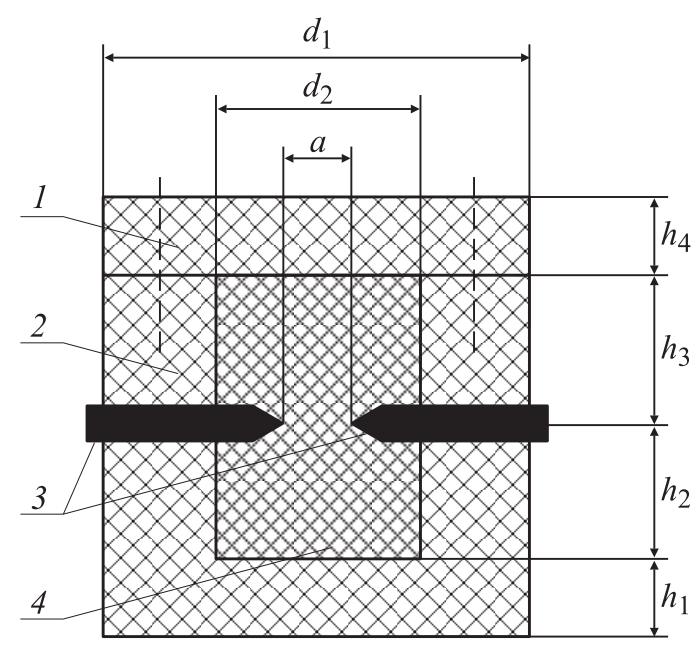

Рис. 1. Контейнер с энергонасыщенной смесью: 1 - крышка, 2 - корпус, 3 - электроды, 4 - энергонасыщенная смесь (штриховыми линиями показаны места крепления крышки).

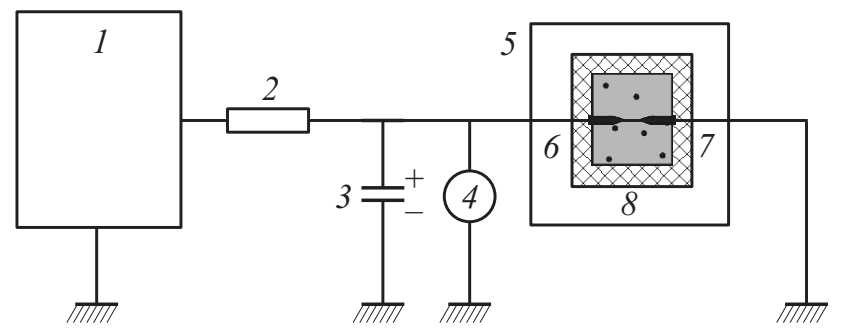

Рис. 2. Электрическая схема проведения эксперимента: 1 - источник высокого напряжения, 2 - зарядное сопротивление, 3 - зарядная емкость $(0.5 \mu \mathrm{F}), 4-$ киловольтметр, 5 - камера, 6 - высоковольтный электрод, 7 - заземленный электрод, 8 - контейнер с энергонасыщенной смесью.

При плавной подаче статического напряжения со скоростью $1000 \mathrm{~V} / \mathrm{s}$ между электродами возникает искровой пробой и происходит возбуждение ВП в энергонасыщенной смеси. В трех случаях, кроме указанной скорости повышения напряжения, были проведены эксперименты по определению напряжения пробоя со скоростью $2000 \mathrm{~V} / \mathrm{s}$. Напряжение пробоя определяли с помощью киловольтметра Инициирование взрывчатых превращений в такой энергонасыщенной композиции, как уже было сказано выше, происходит в результате совместного действия нагрева и ударной волны.

\section{Результаты экспериментов и их обсуждение}

В работе [7] были получены результаты по электрическому пробою в прессованном азиде свинца (типичном инициирующем взрывчатом веществе). На основании этих результатов автор утверждал, что пробой происходит по воздушным каналам между кристаллами вещества. Поэтому в рамках настоящей работы были проведены эксперименты по пробою воздушного промежутка между электродами с целью устранения утверждений о пробое наших композиций и чистых ЭНМ по воздушным каналам между частицами материалов. Другая цель экспериментов по электрическому пробою чистого воздуха - определение характеристик самой установки.

Результаты экспериментов были следующие: напряжение пробоя воздушного промежутка шириной $3 \mathrm{~mm}$ между остроконечными электродами составляет $U_{b}=$ $=8.0-8.4 \mathrm{kV}$. Это несколько меньше, чем следует из расчетно-экспериментальной электрической прочности воздуха [6] $(\sim 10 \mathrm{kV})$ при том же расстоянии между электродами $(3 \mathrm{~mm})$. Различие объясняется тем, что электрическая прочность воздуха определяется с помощью плоских электродов [8], а в наших экспериментах использовались заостренные электроды, что приводило к локальному повышению напряженности электрического поля в зазоре и, как следствие, к снижению напряжения пробоя. 
Таблица 1. Результаты экспериментов с композициями на основе ЭНМ-1

\begin{tabular}{c|c|c|c|c|c|c}
\hline \multirow{2}{*}{ № 0} & \multicolumn{3}{|c|}{ ЭНМ-1 + } & \multicolumn{3}{c}{ ЭНМ-1 + ДНА } \\
\cline { 2 - 7 } & $\% \mathrm{CuO}$ & $\rho, \mathrm{g} / \mathrm{cm}^{3}$ & $U_{\mathrm{br}}, \mathrm{kV}$ & $\%$ ДНА & $\rho, \mathrm{g} / \mathrm{cm}^{3}$ & $U_{\mathrm{br}}, \mathrm{kV}$ \\
\hline 1 & 0 & 0.7 & 9.5 & 0 & 0.7 & 9.5 \\
2 & 4 & 0.75 & 9.0 & 3 & 0.72 & 9.0 \\
3 & 5 & 0.76 & $7.5 / 8.2$ & 4 & 0.72 & 7.7 \\
4 & 6 & 0.8 & $5.0 / 6.6$ & 5 & 0.77 & 7.55 \\
5 & 6.8 & 0.8 & $3.8 / 6.25$ & 7 & 0.78 & 7.0 \\
6 & 7.5 & 0.8 & 0.8 & 12 & 0.78 & \\
7 & 9.5 & 0.85 & 0.5 & 20 & 0.8 & 6.15 \\
8 & 12.5 & 0.87 & 0.35 & 36 & 0.81 & 5.4 \\
9 & 100 & 1.9 & 0.22 & 100 & 0.85 & 4.25
\end{tabular}

Пр и ме чан и е.Приведены средние значения по 3-4 экспериментам (в табл. 2 - аналогично). В знаменателе приведены значения напряжения пробоя при скорости подъема напряжения $2.0 \mathrm{kV} / \mathrm{s}$.

Таблица 2. Результаты экспериментов с композициями ЭНM-2 + $\mathrm{CuO}$

\begin{tabular}{c|c|c|c}
\hline № & \% CuO & $\rho, \mathrm{g} / \mathrm{cm}^{3}$ & $U, \mathrm{kV}$ \\
\hline 1 & 0 & 1.82 & $5.6-6.0$ \\
2 & 1.25 & 1.82 & $3.2-3.6$ \\
3 & 2.5 & 1.84 & $1.5-1.8$ \\
5 & 5.0 & 1.85 & 1.0 \\
6 & 7.5 & 1.86 & 1.0 \\
7 & 10 & 1.87 & 1.0 \\
8 & 12.5 & 1.86 & $0.5-0.7$ \\
9 & 15.0 & 1.86 & $0^{*}$
\end{tabular}

Примечание . * означает отсутствие разряда, ток течет по проводнику.

После этого были проведены эксперименты с ЭНМ-1 и ЭНМ-2 без добавок. Было установлено, что пробой (инициирование) ЭНМ-1 происходит при напряжении $U_{b}=9.4-9.6 \mathrm{kV}$, ЭНМ-2 - при $U_{b}=5.7-5.9 \mathrm{kV}$. Затем были проведены эксперименты с композициями ЭНМ-1 + CuO, ЭНМ-1 + ДНА и ЭНМ- $2+\mathrm{CuO}$. Bo всех экспериментах скорость подъема напряжения $\dot{U}_{b} \approx 1.0 \mathrm{kV} / \mathrm{s}$ (кроме значений, приведенных в знаменателе). Все результаты приведены в табл. 1 и 2.

Во всех экспериментах, где произошел пробой, происходило инициирование энергонасыщенной композиции (или чистых ЭНМ), в образцах происходили некие взрывчатые превращения (горение или взрыв, идентифицировать не удалось), и контейнеры разрушались на множество мелких фрагментов (рис. 3).

Экспериментальные данные также показывают, что добавление в образец любого из рассмотренных нанопорошков приводит к снижению напряжения пробоя, причем тем сильнее, чем больше содержание нанопорошка в смеси. Результаты, приведенные в таблицах, показывают, что разные нанопорошки по-разному снижают напряжение пробоя (инициирования) даже для одного и того же базового порошка (ЭНМ-1). С увеличением содержания

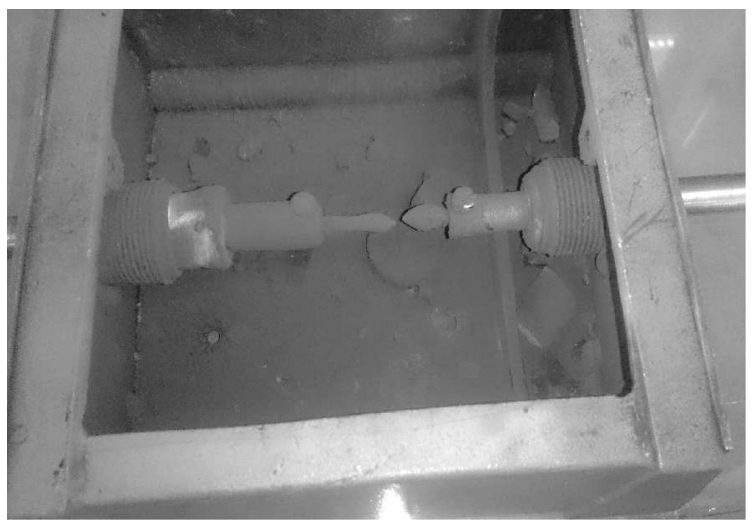

Рис. 3. Разрушенный контейнер в экспериментальной камере.

нанопорошка в смеси (энергонасыщенной композиции) напряжение пробоя (инициирования) асимптотически стремится к некоторому, вообще говоря, ненулевому пределу, который зависит, главным образом, от свойств нанопорошка. Так, напряжения пробоя таких разных энергонасыщенных материалов (один на основе пикрата калия, другой на основе свинцового сурика) при добавлении нанопорошка $\mathrm{CuO}$ стремятся к близким значениям, соответствующим напряжению пробоя чистого нанопорошка меди $(\sim 0.22 \mathrm{kV})$. В то же время напряжение пробоя пикрата калия при добавлении детонационных наноалмазов асимптотически стремится к уровню $\sim 5.0 \mathrm{kV}$, тогда как напряжения пробоя порошка ДНА $-4.25 \mathrm{kV}$. Такое снижение напряжения пробоя и соответственно напряжения инициирования может быть связано с тем, что частицы наноразмерного порошка при определенных условиях образуют перколяционные цепочки, пронизывающие весь образец, по которым может происходить пробой. Так ли это?

\section{Теоретическая часть}

\section{Механизм пробоя порошковой смеси с наноразмерными добавками}

Оценки показывают, что в рассматриваемых порошковых смесях такие цепочки не могут образовываться за счет естественной („геометрической“) перколяции. Действительно, для того, чтобы такие цепочки возникли в объеме пор крупного порошка, объемное содержание наноразмерного порошка в порах крупного порошка должно достигать объемного перколяционного предела [4]. Например, для нанопорошка $\mathrm{CuO}$ объемный перколяционный предел достигается при содержании меди в образце $m \approx 40$ wt.\%. В таких смесях возможна также поверхностная перколяция, когда высокодисперсные частицы добавки располагаются на поверхности зерен основного порошка [4]. В этом случае на поверхности зерен основного порошка могут возникать перколяционные кластеры, пронизывающие весь образец, если 
содержание высокодисперсного порошка превысит предел поверхностной перколяции [4]. Для смеси пикрата калия с нанопорошком $\mathrm{CuO}$ поверхностный перколяционный предел возникает при содержании меди в образце $m \approx 1$ wt.\%. Вместе с тем эксперименты показывают, что заметное снижение напряжения пробоя наблюдается при значительно большем содержании нанопорошка меди в смеси (> 7wt.\%). Это может быть связано с тем, что при включении электрического поля наночастицы, находящиеся на поверхности зерен основного порошка, поляризуются и отрываются от поверхности.

За счет поляризации диэлектрические частицы нанопорошка приобретают дипольные моменты. В результате под действием дипольного притяжения и дипольного взаимодействия с внешним электрическим полем наночастицы будут собираться в кластеры, вытянутые вдоль силовых линий электрического поля. С течением времени размеры этих кластеров будут расти и при определенных условиях в порах образца может возникнуть бесконечный перколяционный кластер, замыкающий электроды. В этом случае в образце возникнет вынужденная (в отличие от естественной „геометрической“') перколяция.

Перколяционные кластеры обладают определенной электрической прочностью. В частности, для каждого типа наночастиц можно ввести напряжение пробоя перколяционного кластера $U_{n}$, которое описывается тепловой моделью [4]. Для оценки напряжения $U_{n}$ можно взять напряжение пробоя чистого нанопорошка. Тогда для нанопорошка меди $U_{n}=0.22 \mathrm{kV}$.

$\mathrm{B}$ представленной серии экспериментов напряжение на электродах монотонно возрастало со временем. Учитывая, что процесс формирования перколяционного кластера также происходит во времени, условия электрического пробоя образца будут зависеть от скорости подъема напряжения. Так, если в момент образования перколяционного кластера напряжение на электродах превышает значение $U_{n}$, то происходит пробой кластера, который повлечет за собой пробой образца. В этом случае напряжением пробоя образца будет напряжение, достигнутое в момент возникновения в системе перколяционного кластера. Если в момент образования перколяционного кластера достигнутое напряжение на электродах меньше $U_{n}$, то кластер является изолятором и пробой в этот момент не происходит; он произойдет при дальнейшем повышении напряжения, когда будет достигнуто напряжение $U_{n}$. В этом случае напряжение пробоя образца будет равно $U_{n}$. Если же между электродами было достигнуто напряжение пробоя воздуха в порах образца $U_{0}$ и при этом перколяционный кластер в системе не успел образоваться, то пробой произойдет при напряжении $U_{0}$.

Таким образом, напряжение пробоя образца лежит в пределах $U_{n} \leq U \leq U_{0}$ и зависит от скорости подъема напряжения и скорости формирования перколяционного кластера в образце.

\section{Вынужденная перколяция наночастиц в электрическом поле}

Рассмотрим поведение диэлектрических наночастиц в электрическом поле. При приложении внешнего электрического поля $\mathbf{E}$ в диэлектрических наночастицах возникает наведенный дипольный момент [9]

$$
\mathbf{p}=V_{n} \frac{\varepsilon_{n}-1}{4 \pi} \mathbf{E}
$$

который будет направлен вдоль вектора электрического поля $\mathbf{E}$.

Здесь $V_{n}$ - объем наночастицы, $\varepsilon_{n}-$ ее диэлектрическая проницаемость, $\mathbf{E}$ - напряженность электрического поля в порах основного порошка (энергонасыщенного материала).

Электрическое поле между электродами для используемой конструкции электродов является неоднородным, особенно вблизи электродов. В результате на каждую поляризованную наночастицу будет действовать сила [9]

$$
\mathbf{F}_{f}=V_{n} \frac{\varepsilon_{n}-1}{8 \pi} \nabla \mathbf{E}^{2},
$$

которая направлена в сторону возрастания модуля вектоpa $\mathbf{E}$, т. е. в сторону ближайшего электрода. Эта сила будет отрывать прилипшие наночастицы от зерен крупного порошка и притягивать их к ближайшему электроду. В результате этого при подаче напряжения на электроды существовавшая в исходном порошке поверхностная перколяция наночастиц исчезнет, и они начнут двигаться в объеме пор.

Кроме этого, между поляризованными наночастицами возникнет дипольное взаимодействие, в результате которого наночастицы будут притягиваться друг к другу. Рассматривая наночастицы как диполи, получим соотношение для силы, действующей на наночастицу $i$ со стороны наночастицы $j[10]$ :

$$
\mathbf{F}_{i j}=-\nabla_{i} \Pi_{i j}
$$

где

$$
\Pi_{i j}=\frac{\left(\mathbf{p}_{i} \mathbf{p}_{j}\right) R_{i j}^{2}-3\left(\mathbf{p}_{i} \mathbf{R}_{i j}\right)\left(\mathbf{p}_{j} \mathbf{R}_{i j}\right)}{R_{i j}^{5}} .
$$

Здесь $\mathbf{R}_{i j}$ - вектор расстояния между частицами $i$ и $j$. Дипольные моменты наночастиц $p_{i}$ и $p_{j}$ определяются соотношением (1).

Выражение для потенциальной энергии (4) можно упростить, если принять, что все наночастицы одинаковые, а также учесть, что дипольное притяжение быстро убывает с расстоянием. В этом случае можно считать, что для всех взаимодействующих частиц поляризующее поле $\mathbf{E}$ является одинаковым.

Тогда, учитывая (1), получим

$$
\Pi_{i j}=V_{n}^{2} \frac{\left(\varepsilon_{n}-1\right)^{2}}{16 \pi^{2}} \frac{\mathbf{E}^{2} R_{i j}^{2}-3\left(\mathbf{E R}_{i j}\right)^{2}}{R_{i j}^{5}} .
$$


Отметим, что при расчете наведенного дипольного момента наночастицы в формулу (1) должна входить напряженность суммарного электростатического поля, включающая в себя как внешнее поле, созданное электродами, так и дипольные поля, создаваемые поляризованными частицами. Однако наведенные дипольные поля являются эффектом второго порядка малости и в дальнейшем под $\mathbf{E}$ будем понимать только поле, созданное в порах порошка без учета наночастиц. Выражение для силы между частицами из (3) и (5) записывается в следующем виде:

$$
\begin{aligned}
\mathbf{F}_{i j} & =V_{n}^{2} \frac{3\left(\varepsilon_{n}-1\right)^{2}}{16 \pi^{2}} \\
& \times \frac{\mathbf{R}_{i j}\left(\mathbf{E}^{2} R_{i j}^{2}+5\left(\mathbf{E} \mathbf{R}_{i j}\right)^{2}\right)+2 \mathbf{E}\left(\mathbf{E} R_{i j}\right) R_{i j}^{2}}{R_{i j}^{7}} .
\end{aligned}
$$

Уравнение движения наночастиц в порах крупного порошка имеет вид

$$
m \frac{\boldsymbol{\vartheta}_{i}}{d t}=\mathbf{F}_{f i}+\sum_{i \neq j} \mathbf{F}_{i j}-\beta^{-1} \boldsymbol{\vartheta}_{i},
$$

где последнее слагаемое описывает силу сопротивления, которую испытывает наночастица при движении в воздухе; $\beta^{-1}$ - коэффициент сопротивления.

Рассматривая медленные движения наночастиц в воздухе (соответствующие малым числам Рейнольдса), можно пренебречь инерционной силой по сравнению с силой сопротивления. В результате получим сверхзатухающее приближение уравнения (7) [11]

$$
\beta \boldsymbol{\vartheta}_{i}=F_{f i}+\sum_{i \neq j} F_{i j}
$$

или

$$
\frac{d \mathbf{r}_{i}}{d t}=\beta\left(\dot{\mathbf{F}}_{f}+\sum_{i \neq j} F_{i j}\right) .
$$

В этом приближении коэффициент сопротивления $\beta^{-1}$ пропорционален характерному размеру наночастиц и вязкости воздуха и для рассматриваемой системы он будет считаться постоянным.

К уравнению (9) добавляются связи, ограничивающие область движения наночастиц поверхностью зерен крупного порошка, а также связи, исключающие взаимное проникновение притягивающихся наночастиц. Формально эти связи можно задать в виде отталкивающих сил, которые возникают, если две частицы начинают пересекаться [11].

Учитывая уравнения (2) и (6), уравнение (9) можно привести к виду

$$
\begin{aligned}
\frac{d \mathbf{r}_{i}}{d t} & =\beta\left(V_{n} \frac{\varepsilon_{n}-1}{8 \pi} \nabla_{i} \mathbf{E}^{2}+V_{n}^{2} \frac{3\left(\varepsilon_{n}-1\right)^{2}}{16 \pi^{2}}\right. \\
& \left.\times \sum_{i \neq j} \frac{\mathbf{R}_{i j}\left(\mathbf{E}^{2} R_{i j}^{2}+5\left(\mathbf{E R}_{i j}\right)^{2}\right)+2 \mathbf{E}\left(\mathbf{E R}_{i j}\right) R_{i j}^{2}}{R_{i j}^{7}}\right) .
\end{aligned}
$$

Напряженность электрического поля в порах энергонасыщенной композиции можно определить, исходя из следующих положений. Из оображений размерности напряженность внешнего электрического поля $\mathbf{E}_{S}$, создаваемого электродами,

$$
\mathbf{E}_{S}=\gamma U / a,
$$

где $\gamma$ - безразмерный параметр, зависящий от формы и размеров электродов и не зависящий от свойств окружающей среды.

Для оценки электрического поля в порах порошка примем, что поры являются сферическими включениями в непрерывной диэлектрической среде, которой является исследуемый порошок. Электрическое поле внутри одной изолированной сферической поры в сплошной диэлектрической среде определяется выражением [9]

$$
\mathbf{E}=\left(3 \varepsilon_{p} /\left(2 \varepsilon_{p}+\varepsilon_{0}\right)\right) \mathbf{E}_{S} .
$$

Здесь $\varepsilon_{p}$ - диэлектрическая проницаемость среды, окружающей пору, $\varepsilon_{0} \approx 1-$ то же самое для воздуха, заполняющего поры. В случае, когда имеется множество пор, формулу (12) можно переписать в виде

$$
\mathbf{E}=\left(3 \alpha \varepsilon_{p} /\left(2 \varepsilon_{p}+1\right)\right) \mathbf{E}_{S}
$$

где $\alpha \sim 1-$ коэффициент, учитывающий взаимное влияние пор.

С учетом (11) и (13) напряженность электрического поля в порах порошка определяется выражением

$$
\mathbf{E}=\left(3 \gamma \alpha \varepsilon_{p} /\left(2 \varepsilon_{p}+1\right)\right)(U / a) .
$$

Пусть пробой чистого порошка (без добавок) происходит при напряжении на электродах, равном $U_{0}$, которое определяется соотношением

$$
U_{0}=\left(3 \gamma \alpha \varepsilon_{p} /\left(2 \varepsilon_{p}+1\right)\right)\left(E_{\mathrm{cr}} a\right),
$$

где $E_{\mathrm{cr}}-$ напряженность электрического поля внутри пор, при которой наступает пробой порошка.

С учетом (14) и (15) напряженность электрического поля в порах при произвольном напряжении между электродами $U$ определяется выражением

$$
\mathbf{E}=E_{\mathrm{cr}}\left(U / U_{0}\right)
$$

Соотношение (16) показывает, что чем меньше напряжение пробоя чистого порошка, тем больше будет напряженность электрического поля в его порах при заданном напряжении на электродах. Для энергонасыщенной композиции (смеси ЭНМ с наноразмерными добавками) выражение (16) можно записать в следующем виде:

$$
\mathbf{E}=\mathbf{h}(r / a) E_{\mathrm{cr}}\left(U / U_{0}\right)
$$

где $\mathbf{E}=\mathbf{h}(r / a)$ - безразмерная функция, слабо зависящая от свойств порошка и наноразмерной добавки. 


$$
\begin{aligned}
& \text { Подстановка (17) в }(10) \\
& \frac{d \mathbf{r}_{i}}{d t}=\beta E_{\mathrm{cr}}^{2}\left(U / U_{0}\right)^{2}\left(V_{n} \frac{\varepsilon_{n}-1}{8 \pi} \nabla_{i} \mathbf{h}^{2}+V_{n}^{2} \frac{3\left(\varepsilon_{n}-1\right)^{2}}{16 \pi^{2}}\right. \\
& \left.\times \sum_{i \neq j} \frac{\mathbf{R}_{i j}\left(\mathbf{h}^{2} R_{i j}^{2}+5\left(\mathbf{h R}_{i j}\right)^{2}\right)+2 \mathbf{h}\left(\mathbf{h} \mathbf{R}_{i j}\right) R_{i j}^{2}}{R_{i j}^{7}}\right) .
\end{aligned}
$$

Так как в экспериментах напряжение монотонно возрастало с течением времени, можно вместо дифференцирования по времени ввести дифференцирование по напряжению, т. е. $\frac{d}{d t}=\dot{U} \frac{d}{d U}$, здесь $\dot{U}-$ скорость изменения напряжения. Тогда уравнение (18) записывается в следующем виде:

$$
\begin{aligned}
& \frac{d \mathbf{r}_{i}}{d z}=\beta E_{\mathrm{cr}}^{2}\left(\frac{1}{U_{0}^{2}}\right)\left(V_{n} \frac{\varepsilon_{n}-1}{8 \pi} \nabla_{i} \mathbf{h}^{2}+V_{n}^{2} \frac{3\left(\varepsilon_{n}-1\right)^{2}}{16 \pi^{2}}\right. \\
& \left.\times \sum_{i \neq j} \frac{\mathbf{R}_{i j}\left(\mathbf{h}^{2} R_{i j}^{2}+5\left(\mathbf{h} \mathbf{R}_{i j}\right)^{2}\right)+2 \mathbf{h}\left(\mathbf{h} \mathbf{R}_{i j}\right) R_{i j}^{2}}{R_{i j}^{7}}\right),
\end{aligned}
$$

где $z=\int_{0}^{U}\left(U^{2} / U_{0}\right) d U$.

В общем случае скорость изменения напряжения $\dot{U}$ может меняться с изменением напряжения и времени. Если же скорость изменения напряжения постоянна $(\dot{U}=$ const $)$, то $z=U^{3} / 3 U_{0}$. Из уравнения (19) следует, что его правая часть не зависит от напряжения, а определяется только мгновенным расположением наночастиц в порах.

Уравнения (19) для всех наночастиц $i=1,2, \ldots$ с учетом связей образует замкнутую систему, которая может быть решена численно. Под действием сил наночастицы согласованно двигаются в сторону электродов. При обеспечении совместных смещений наночастицы притягиваются друг к другу и образуют цепочки (кластеры), вытянутые вдоль силовых линий поля Е. С течением времени длина таких цепочек будет увеличиваться и в какой-то момент времени возникнет бесконечный перколяционный кластер из наночастиц, который будет пронизывать всю систему от одного электрода до другого.

\section{Модель пробоя порошковой композиции с наноразмерными добавками}

С учетом условий электрического пробоя композиции из энергонасыщенного порошкового материала и наноразмерных добавок, сформулированных в подразделе „Механизм пробоя...“ и результатов предыдущего раздела, можно записать соотношение, которое определяет напряжение пробоя порошковой смеси:

$$
U_{b}=\left\{\begin{array}{l}
U_{n}, U_{p<U_{n},} \\
U_{p}, U_{n}<U_{p}<U_{0}, \\
U_{0}, U_{p}>U_{0},
\end{array}\right.
$$

где $U_{p}$ - напряжение между электродами, достигаемое в момент образования перколяционного кластера.
Момент образования перколяционного кластера из наночастиц (и соответствующее этому моменту напряжение) может быть найден численным решением системы уравнений (19). Однако анализ этой системы позволяет сделать ряд выводов без ее решения. Для этого запишем систему уравнений (19) в безразмерном виде.

Рассматриваемая система имеет четыре характерных пространственных масштаба: среднее расстояние между наночастицами в порах $l$, характерный размер пор $\delta$ (он имеет порядок среднего размера зерен энергонасыщенного материала), внешний размер системы расстояние между электродами $a$ и характерный размер наночастиц $d_{n}$. Эти масштабы связаны естественными соотношениями:

$$
d_{n} \ll l<\delta \ll a .
$$

Все эти характерные размеры в той или иной мере должны входить в решение системы уравнений (19). Можно полагать, что при совместимости и согласованности смещений наночастиц формирование перколяционного кластера в каждой поре будет происходить синхронно. Поэтому приближенно можно считать, что моментом образования бесконечного кластера является момент формирования перколяционного кластера в характерной поре. Такое приближение позволяет рассматривать соотношение (19) для отдельной поры, и поскольку $\delta / a \ll 1$, то в пределах одной поры безразмерные векторы $\mathbf{h}$ и $\nabla_{i} \mathbf{h}^{2}$ могут считаться постоянными. Для дальнейшего анализа оценим масштабы разных членов правой части уравнения (19).

Расстояние между частицами $\mathbf{R}_{i j}$, а также сами координаты частиц имеют характерный размер (масштаб) $l$, который определяется из соотношения

$$
\frac{V_{n}}{l^{3}}=\frac{m}{1-m} \frac{\rho}{1-\rho} \frac{\rho_{n}}{\rho_{c}}
$$

где $\rho-$ относительная плотность энергонасыщенного (крупного) порошка в композиции, $\rho_{n}$ и $\rho_{c}-$ плотности материалов наночастиц и ЭНМ соответственно, $m-$ массовая доля наноразмерного порошка в композиции.

Перепишем уравнение (19) в безразмерном виде

$$
\begin{aligned}
& \frac{d \mathbf{r}_{i}}{d \tau}=\left(\frac{l^{4}}{8 \pi a V_{n}\left(\varepsilon_{n}-1\right)}\right) \nabla_{i} \mathbf{h}^{2}+\frac{3}{16 \pi^{2}} \\
& \times \sum_{j \neq i} \frac{\mathbf{R}_{i j}\left(\mathbf{h}^{2} R_{i j}^{2}+5\left(\mathbf{h} \mathbf{R}_{i j}\right)^{2}\right)+2 \mathbf{h}\left(\mathbf{h} \mathbf{R}_{i j}\right) R_{i j}^{2}}{R_{i j}^{7}},
\end{aligned}
$$

где $\tau=\beta\left(\frac{E_{\text {cr }}}{U_{0}\left(\varepsilon_{n}-1\right)}\right)^{2} \frac{V_{n}^{2}}{l^{5}} z-$ безразмерный параметр. За исключением безразмерного множителя $l^{4} / a V_{n}$, все остальные члены в правой части уравнения (22) не зависят от масштаба $l$. Размеры пор $\delta$ в решении системы уравнений (22) возникнут в результате учета связей: движение наночастиц в электростатическом поле ограничено поверхностью зерен крупных частиц ЭНМ. 
Наиболее медленно образование кластеров будет происходить в середине зазора между электродами, так как в этой точке электростатическое поле имеет наименьшую напряженность. Поэтому можно считать, что если перколяционный кластер образовался в порах, расположенных в центре зазора между электродами, то он образовался и в остальных порах. Но в центре зазора в силу симметрии $\nabla_{i} \mathbf{h}^{2} \approx 0$, т.е. в этом месте формирование перколяционного кластера будет происходить, главным образом, за счет дипольного притяжения поляризованных наночастиц.

В этом случае уравнение (21) можно переписать в виде

$$
\begin{aligned}
\frac{d \mathbf{r}_{i}}{d \tau} & =\frac{3}{16 \pi^{2}} \\
& \times \sum_{j \neq i} \frac{\mathbf{R}_{i j}\left(\mathbf{h}^{2} R_{i j}^{2}+5\left(\mathbf{h} \mathbf{R}_{i j}\right)^{2}\right)+2 \mathbf{h}\left(\mathbf{h} \mathbf{R}_{i j}\right) R_{i j}^{2}}{R_{i j}^{7}},
\end{aligned}
$$

в котором правая часть не зависит от масштаба $l$ и от свойств наноразмерного порошка. Однако в целом решение системы (23) будет зависеть от отношения $l / \delta$, поскольку размер области движения наночастиц ограничен размерами пор в порошке ЭНМ. Но роль размеров пор будет заметна только при очень малом содержании наноразмерного порошка в композиции, т.е. только тогда, когда для образования перколяционного кластера в поре необходимо собрать вместе все наночастицы, находящиеся в поре. При относительно большом содержании наночастиц в смеси в каждой поре будут формироваться одновременно несколько кластеров, в которые будут собираться только ближайшие между собой наночастицы. В этом случае размер пор $\delta$ не будет играть заметной роли и можно полагать, что решение системы уравнений (23) будет универсальной функцией, одинаковой для любых смесей и любых концентраций наноразмерного порошка. Это означает, что формирование перколяционного кластера будет происходить при одном и том же значении безразмерного параметра $\tau=\tau_{0}$, т. е.

$$
\tau_{0}=\beta\left(\frac{E_{\mathrm{cr}}}{U_{0}\left(\varepsilon_{n}-1\right)}\right) \frac{V_{n}^{2}}{l^{5}} z .
$$

Подставляя в (24) значение $z$, с учетом (21) получим выражение для определения напряжения пробоя (и соответственно инициирования) энергонасыщенной композиции

$$
U_{p}=A U_{0}^{2 / 3} \dot{U}^{1 / 3}\left(\frac{1-m}{m}\right)^{5 / 9}
$$

где

$$
\begin{gathered}
A=A_{0}\left(\varepsilon_{n}-1\right)^{2 / 3}\left(\frac{\rho_{c}}{\rho_{n}} \frac{1-\rho}{\rho}\right)^{5 / 9}, \\
A_{0}=\left(\frac{3 \tau_{0}}{\beta E_{\mathrm{cr}}^{2} V_{n}^{1 / 3}}\right)^{1 / 3}-\text { постоянная. }
\end{gathered}
$$

Поскольку для медленных движений наноразмерных частиц в воздухе $\beta \sim V_{n}^{-1 / 3}$, то можно полагать, что постоянная $A_{0}$ не зависит от свойств испытуемой энергонасыщенной композиции (т. е. не зависит ни от ЭНМ, ни от наноразмерного порошка, ни от его концентрации). Однако $A_{0}$, возможно, слабо зависит от конструктивных параметров экспериментальной установки, влияющих на однородность/неоднородность электрического поля между электродами.

Параметр $A$ (26) определяет зависимость напряжения пробоя от диэлектрической проницаемости наноразмерного порошка, от плотностей ЭНМ и нанопорошка и от относительной плотности ЭНМ в энергонасыщенной композиции. Согласно (26), параметр $A$ будет изменяться при изменении ЭНМ и наноразмерного порошка в композиции.

Соотношение (25) определяет зависимость напряжения пробоя (инициирования) энергонасыщенной композиции, содержащей наночастицы, от их массовой доли в смеси $m$, от напряжения пробоя чистого ЭНМ без наноразмерных добавок $U_{0}$ и от скорости подъема напряжения в эксперименте. Так, увеличение скорости подъема напряжения $\dot{U}$ в два раза теоретически должно приводить к увеличению напряжения пробоя экспериментальной композиции на 26\%. Экспериментальные данные, приведенные в табл. 1, несмотря на ограниченность интервала содержания $\mathrm{CuO}$, по крайней мере, качественно подтверждают данный вывод. Только качественное, а не количественное согласие с экспериментом (с точки зрения скорости подъема напряжения) указывает на приближенность соотношения (25). Также с учетом неопределенности многих параметров, входящих в коэффициент $A(26)$, его можно считать только коэффициентом согласования.

На рис. 4 приведены рассчитанные по зависимостям (20) и (25) кривые и нанесены экспериментальные данные для испытанных энергонасыщенных композиций. Из приведенных расчетных и экспериментальных результатов следует, что теоретическая зависимость удовлетворительно описывает данные экспериментов по инициированию композиций ЭНМ-2 + $\mathrm{CuO}$, ЭНМ-1 + ДНА (рис. 4, $b$ ) и качественно описывает ЭНМ-1 + СuО при $\dot{U}=2000 \mathrm{~V} / \mathrm{s}$ (рис. 4, $a$ ). Вместе с тем теоретическая зависимость (20), (25) достаточно плохо описывает эксперименты по инициированию композиции ЭНМ-1 + CuO. С чем это может быть связано?

При выводе формулы (24) и расчета по ней напряжения пробоя (инициирования) предполагалось, что в формировании перколяционного кластера участвуют все наноразмерные частицы, находящиеся в порошковой композиции. Однако в действительности часть наночастиц может быть связана за счет электростатического прилипания некоторых частиц к поверхности крупных зерен ЭНМ. Именно такие наночастицы и не будут участвовать в формировании перколяционного кластера в объеме пор. Следовательно, в расчетах по пробою 

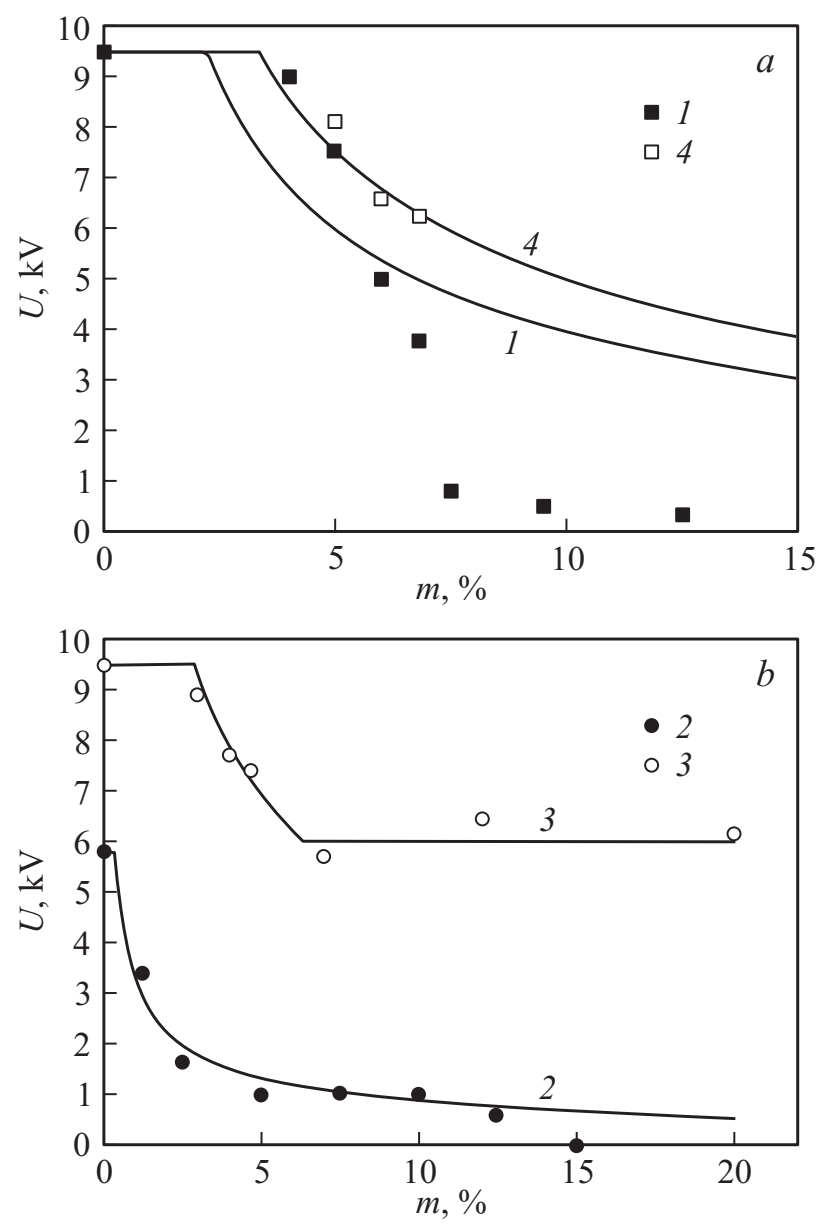

Рис. 4. Сравнение экспериментальных и теоретической зависимостей напряжения пробоя (инициирования) порошковых смесей от содержания в них наноразмерных добавок: 1 и 4 - ЭНМ-1 + CuO; 2 - ЭНМ-2 + CuO; 3 - ЭНМ-1 + ДНА; данные 1-3 соответствуют скорости подъема напряжения в экспериментах $\dot{U}=1.0 \mathrm{kV} / \mathrm{s}$; данные 4 соответствуют скорости подъема напряжения $\dot{U}=2.0 \mathrm{kV} / \mathrm{s}$. Линии 1 и $4-A=0.26$; линия $2-A=0.08$; линия $3-A=0.3$.

композиции ЭНМ-1 + $\mathrm{CuO}$ необходимо уменьшить количество наночастиц в объеме пор на количество частиц, прилипших к поверхности зерен энергонасыщенного материала. Необходимо отметить, что для других использованных добавок заметного прилипания наночастиц к поверхности зерен не наблюдалось. Вероятно, именно этим и объясняется, что экспериментальные данные для остальных композиций удовлетворительно описываются теоретической зависимостью (20), (25), которая не учитывает прилипание наночастиц к поверхности зерен основного порошка. Заметим, что прилипание наночастиц к поверхности зерен ЭНМ-1 (на основе пикрата калия) свидетельствует об электрической активности поверхности этих зерен, т.е. о склонности к поляризации. В свою очередь, это может коррелировать с тем, что напряжение пробоя чистого (без добавок) ЭНМ-1 выше, чем напряжение пробоя чистого воздуха в тех же условиях, поскольку электрическая активность поверхности зерен пикрата калия приводит к захвату электронов этой поверхностью.

Исходя из этих предположений, обозначим массовую долю связанных наночастиц $m_{0}$, которую приближенно будем считать постоянной характеристикой порошковой композиции. Если содержание наночастиц в композиции меньше $m_{0}$, то все наночастицы оказываются связанными и не участвуют в формировании перколяционного кластера, т.е. это равносильно полному отсутствию в композиции наночастиц. Если же содержание $\mathrm{CuO}$ в композиции больше $m_{0}$, то в формировании кластеров из наночастиц принимает только часть из наночастиц, массовая доля которых в смеси $m_{1}=m-m_{0}$.

В этом случае формула (25) приобретает вид

$$
U_{p}=A U_{0}^{2 / 3} \dot{U}^{1 / 3}\left(\frac{1-m_{1}}{m_{1}}\right)^{5 / 9},
$$

где $m_{1}$ - массовая доля свободных наночастиц в смеси, которая определяется соотношением

$$
m_{1}=\left\{\begin{array}{l}
0, m \leq m_{0}, \\
m-m_{0}, m>m_{0} .
\end{array}\right.
$$

На рис. 5 приведены зависимости (20), (27) и (28) для порошковой композиции ЭНМ-1 + $\mathrm{CuO}$ при скорости подъема напряжения $\dot{U}=1.0 \mathrm{kV} / \mathrm{s}$ (кривая 1 ) и при скорости $\dot{U}=2.0 \mathrm{kV} / \mathrm{s}$ (кривая 4 ). Из сравнения рис. $4, a$ и 5 видно, что полученные зависимости (20), (27) и (28) значительно лучше описывают экспериментальные данные для $\dot{U}=2.0 \mathrm{kV} / \mathrm{s}$ и незначительно лучше для скорости $\dot{U}=1.0 \mathrm{kV} / \mathrm{s}$ (кривые 2 и 3 на рис. 5 не приведены, поскольку они полностью совпадают с аналогичными кривыми на рис. 4). Можно отметить, что для скорости подъема напряжения $\dot{U}=1.0 \mathrm{kV} / \mathrm{s}$ наблюдается удовлетворительное совпадение теоретических зависимостей

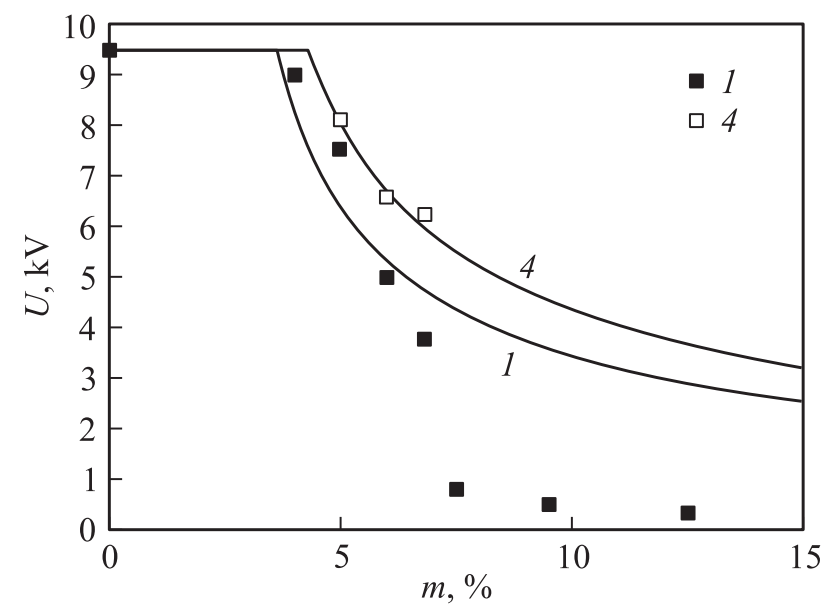

Рис. 5. Сравнение экспериментальных и теоретических зависимостей напряжения пробоя (инициирования) порошковых смесей от содержания в них наноразмерных добавок: нумерация та же, что и на рис. 4. Линии 1 и $4-A=0.2 ; m_{0}=2.3 \%$. 
и экспериментальных данных только для относительно малого содержания наночастиц $(m<6.5 \%)$.

Анализ экспериментальных данных по инициированию композиции ЭНМ-1 $+\mathrm{CuO}$ показывает, что для $m<6.5 \%$ происходит монотонное уменьшение напряжения пробоя (инициирования) с ростом содержания $\mathrm{CuO}$ в композиции, а при $m \approx 6.5 \%$ происходит резкое падение напряжения пробоя до значений, близких к напряжению пробоя порошка $\mathrm{CuO}$. При дальнейшем увеличении доли $\mathrm{CuO}$ в композиции ЭНМ-1+CuO напряжение пробоя изменяется достаточно медленно. То есть по какой-то не очень ясной причине при содержании наночастиц $\mathrm{CuO} m>6.5 \%$ перколяционный кластер образуется очень быстро уже при достаточно малых напряжениях. Одной из таких причин может быть, например, отрыв слоя наночастиц от поверхности зерен пикрата калия, происходящий при низких напряжениях и при превышении содержания наночастиц в смеси выше некоторого критического значения.

\section{Заключение и выводы}

В результате проведенных экспериментальных исследований по инициированию энергонасыщенных материалов с наноразмерными добавками высоковольтным электрическим разрядом установлено.

1. Электрический пробой порошковой композиции является первичным явлением, а инициирование энергонасыщенной смеси является следствием этого пробоя.

2.При добавлении в энергонасыщенные материалы наноразмерных добавок (нанопорошка с окисной пленкой на поверхности и детонационных наноалмазов) наблюдается существенное снижение напряжения пробоя (инициирования), причем тем больше, чем больше содержание в нем наноразмерной добавки.

3. Чем больше скорость подъема напряжения в эксперименте, тем больше напряжение пробоя зарядов ЭНМ с наноразмерными добавками.

На основе полученных экспериментальных результатов предложены:

1. Модель электрического пробоя порошковых смесей, согласно которой снижение напряжения пробоя ЭНМ с наноразмерными добавками связано с образованием бесконечного перколяционного кластера из наночастиц, пронизывающего весь образец. Бесконечный кластер имеет меньшую электрическую прочность, чем воздух, заполняющий поры между частицами энергонасыщенного материала. Данная перколяция является вынужденной и происходит за счет дипольного притяжения наночастиц, электризованных в электростатическом поле электродов. Зависимость напряжения пробоя зарядов от содержания в них нанодобавки связана с инерционностью процесса образования перколяционных кластеров из наночастиц и особенностями проведения экспериментов, в которых напряжение монотонно поднималось с почти постоянной скоростью. В результате напряжением пробоя было то напряжение, которое было достигнуто в момент образования перколяционного кластера.

2. Критериальные зависимости, описывающие изменение напряжения пробоя от содержания в образце наноразмерной добавки. Полученные зависимости согласуются с имеющимися экспериментальными данными.

\section{Список литературы}

[1] Даниленко В.В. Взрыв: физика, техника, технология. М.: Энергоатомиздат, 2010. $784 \mathrm{c.}$

[2] Физика взрыва / Под ред. К.П. Станюковича. М.: Наука, 1975. $704 \mathrm{c}$.

[3] Брагин В.А., Душенок С.А., Куликов В.Г., Савенков Г.Г.идр. // Химическая физика. 2012. Т. 31. № 5. C. 57-64.

[4] Рашковский С.А., Савенков Г.Г. // ЖТФ. 2013. Т. 83. Вып. 4. C. $47-58$.

[5] Лукин А.А., Морозов В.А., Рашковский С.А., Савенков Г.Г. // Химическая физика. 2015. Т. 31. № 4. С. 69-75.

[6] Рашковский С.А., Савенков Г.Г. // Успехи в специальной химии и химической технологии. Труды Всерос. научно-технической конф., посвященной 80-летию основания инженерно-технологического факультета РХТУ им. Д.И. Менделеева. М.: ДеЛи плюс, 2015. С. 261-265.

[7] Стеньгач B.B. // Прикладная механика и техническая физика. 1972. № 1. С. 128-132.

[8] Богородицкий Н.П., Пасынков В.В., Тареев Б.М. Электротехнические материалы. М.: Энергоатомиздат, 1985. 304 с.

[9] Ландау Л.Д., Лифиии, Е.М. Электродинамика сплошных сред. М.: Наука, 1982. 621 с.

[10] Ландау Л.Д., Лифиии, Е.М. Теория поля. М.: Наука, 1973. $504 \mathrm{c}$.

[11] Рашковский C.A. // Физика горения и взрыва. 1999. Т. 35. № 5. C. 65-74. 